$\mathrm{p}=0.0032$ ), 5 out of 7 patients with renal involvement in the rituximab group were eventually hemodialysis dependent.

Conclusions In summary, our data indicated that add-on rituximab in the background of conventional therapy may improve the overall but not the renal survival in SLE-TTP patients.

\section{AGE ADJUSTED CHARLSON'S COMORBIDITY INDEX SCORE PREDICT MAJOR ADVERSE CARDIOVASCULAR EVENTS IN SYSTEMIC LUPUS ERYTHEMOATOUS}

YF Wang. Buddhist Dalin Tzu Chi Hospital, Department of Nuclear Medicine, Chiayi, Taiwan R.O.C.

\subsection{6/lupus-2017-000215.199}

Background and aims Cardiovascular events are the leading cause of death or disability in people with systemic lupus erythematosus (SLE). However, the real mechanism that causes the risk increments for the major adverse cardiovascular events (MACEs) is still not fully understood. The Age-Adjusted Charlson Comorbidity index (ACCI) score is a prognostic classification that was initially developed for a patient who may have a range of co-morbid conditions and has been validated in many clinical settings.

Methods The data for this study were collected from Taiwan's National Health Institute Research Database (NHIRD) for the years 2004 to 2007, 5998 participants were enrolled. All patients' sequential clinically diagnosed SLE data were reviewed from 2002 to 2009 in order to determine their MACEs risk by using ACCI score.

Results Cox proportional hazard ratio model showed that AAC score as a continuous variable conferred $25 \%$ increased risk of MACE in average for each AAC point starting from zero. And when patients were divided into different groups by AAC scores, patients with AAC score more than 6 conferred an adjusted hazard ratio 4.88 (95\% CI 3.84-6.19, p<0.001), compared to those with AAC score 0-1.

Conclusions This is a national cohort study for the evaluation of MACEs for SLE patients. Our results demonstrated that SLE patients with higher ACCI score show significantly higher risk of cardiovascular events and the ACCI score could be applied as an index for MACEs evaluation.

\section{PAN-DYSAUTONOMIA IN SYSTEMIC LUPUS ERYTHEMATOUS: TWO CASES OF SUCCESSFUL TREATMENT AND A REVIEW OF LITERATURE}

${ }^{1} Y$ Yang ${ }^{*},{ }^{2} Y$ Jiang, ${ }^{2} \mathrm{Q}$ Wang. ${ }^{1}$ Peking Union Medical College Hospital, Internal Medicine, Beijing, China; ${ }^{2}$ Peking Union Medical College Hospital, Rheumatology, Beijing, China

\subsection{6/lupus-2017-000215.200}

Background and aims Pan-dysautonomia hasn't been well studied in patients with systemic lupus erythematous (SLE) and the optimal treatments are still to be determined. We report two cases of SLE with pan-dysautonomia and a review of the literature.

Methods Two cases with SLE complicated with pan-dysautonomia were carefully documented and described. Medline, EMBASE, Web of Science and PubMed were searched and 8 other cases were reviewed.

Results Case 1: A 23 year-old female SLE patient with central and peripheral nervous system (CNS and PNS) involvements for 5 years, presented pan-dysautonomia, including severe orthostatic hypotension, dysuria and gastro-intestinal psedoobstruction. Acetylcholine-receptors (AChR) antibody was detected in her cerebrospinal fluid (CSF). After treating with prednisone, tacrolimus, midodrine and fludrocortisone, her symptoms gradually improved in 12 months. Case 2: A 44 year-old female presented with pan-dysautonomia, anti-nuclear and anti-Ro antibody positivity and hypocomplementemia. AChR antibody titer also elevated in her CSF. After similar treatment, and additional plasma exchange for 6 times, all symptoms improved in 3 months. Comblined with other 8 reported cased, 9 were female, 4 and 3 cases complicated with CNS and PNS involvement respectively, 3 cases had AChR antibody detected and all were postive, 4 cases received methylprednisolone pulse therapy. Cyclophosphamide $(n=3)$, azathiopurine $(n=3)$, and tacrolimus (our case 1) were also used. Plasma exchange was applied in our case 2.

Conclusions Pan-dysautonomia can be an initial symptom of SLE. AchR antibody may be useful in early recognising this rare disease. Plasma exchange along with prompt immunosuppressive therapy seem to lead to an early remission of the disease.

Abstract 200 Table 1 A review of 10 SLE cases complicated with pan-dysautonomia.

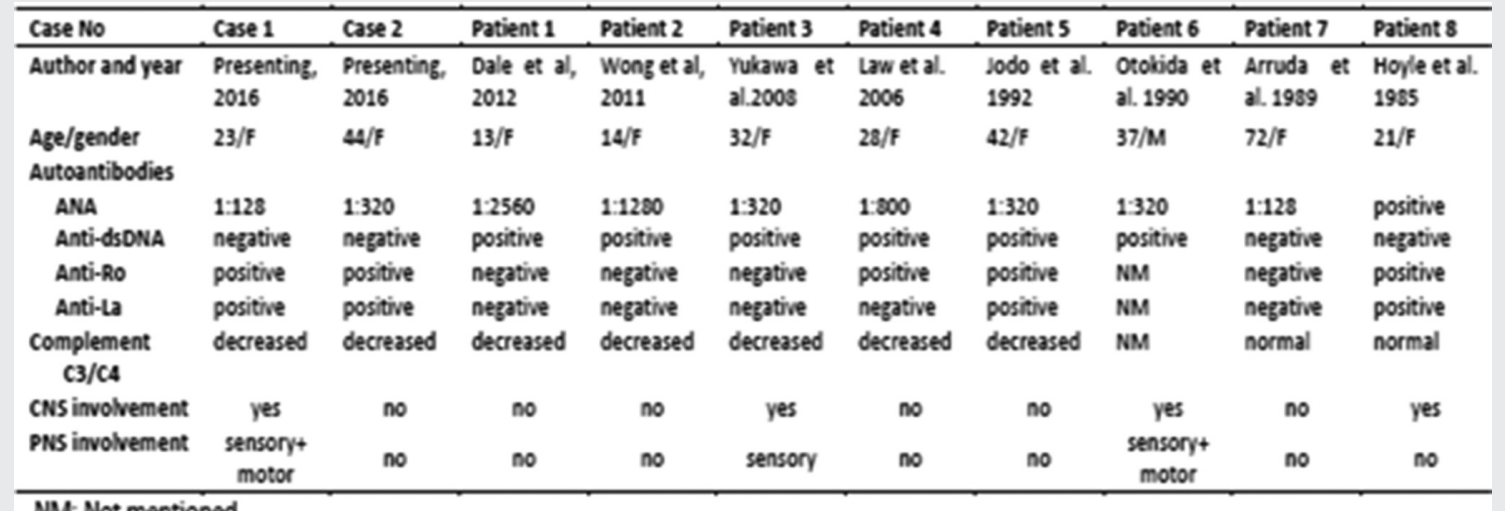

NM: Not mentioned 